\title{
Effect of carbon nanofillers on flexible polyurethane foaming from a chemical and physical perspective
}

\author{
M. Mar Bernal, ${ }^{\star a}$ Samuel Pardo-Alonso, ${ }^{\text {b }}$ Eusebio Solórzano, ${ }^{\mathrm{b}}$ Miguel Ángel Lopez- \\ Manchado, ${ }^{a}$ Raquel Verdejo ${ }^{\mathrm{a}}$ and Miguel Ángel Rodriguez-Perez ${ }^{\mathrm{b}}$
}

\begin{abstract}
The effect of carbon nanoparticles (CNPs) on the physical and chemical events taking place during the foaming evolution of flexible polyurethane (FPU) foams is analysed by in situ X-ray time-resolved imaging. The differences observed in the cellular structure and density evolution of nanocomposite foams are explained in terms of the type of nanoparticles and the functional groups on their surface. The presence of certain types of particles enhanced the bubble nucleation at the beginning of the process although some others did not. The chemical interaction seems to produce delays in the blowing reaction process and promotes coalescence events during foam evolution as regarding the cell density results obtained. This study on the kinetics of polymerisation and morphology development of reactive PU nanocomposite foams contributes to understanding the physical phenomena occurring as a consequence of the CNP-FPU chemical interaction.
\end{abstract}

Received 6th January 2014

Accepted 24th April 2014

DOI: $10.1039 / \mathrm{c} 4 \mathrm{ra00116h}$

www.rsc.org/advances

blowing reaction and produces urea hard segments (HS) and

\section{Introduction}

Polymer nanocomposite foams are receiving increasing attention since the synergy between foams and nanoparticle attributes results in multifunctional and lightweight materials that can find interesting applications at an industrial level. ${ }^{1-3}$ The nanofillers have the ability to reinforce the fine cellular structure of polymeric foams enhancing their mechanical strength, electrical conductivity, nanoscale surface features and biocompatibility. Nevertheless, the properties of the nanocomposite foams are also determined by their final cellular structure, which strongly depends on the nanoparticles type and their interaction with polymer matrix during foaming.

In this field, one of the most interesting polymer foams is reactive polyurethane (PU) foam in congruence with its industrial relevance. In this particular case, the nanofillers are expected to have important effects during the simultaneous interplay of chemical and physical processes, i.e. the polymerisation and foaming of a block copolymer. ${ }^{4-6}$ PU foams are essentially produced by two exothermic reactions between the isocyanate with the hydrogen-active groups of polyol and with water. The reaction between the isocyanate and the polyol is called the gelling reaction and forms urethane linkages. Likewise, the reaction between the isocyanate and water is called the

anstitute of Polymer Science and Technology, CSIC, 28006, Madrid, Spain. E-mail: mar.bernal@imdea.org

${ }^{b}$ CellMat Laboratory, Condensed Matter Physics Department, Science Faculty, University of Valladolid, Paseo de Belén, 7, 47011-Valladolid, Spain. E-mail: samuelpardo.alonso@fmc.uva.es carbon dioxide $\left(\mathrm{CO}_{2}\right)$ gas. ${ }^{7-11}$ Hence, $\mathrm{PU}$ foaming is a balance process between the gas formation and expansion and the viscosity increase occurring during the polymerisation. The presence of any additional substance, from surfactants to fillers, may intervene in this subtle balance promoting or holding back the reaction. Therefore, real-time monitoring of these systems is an essential study to understand nanoparticlePU interactions during the reactive foaming process.

Different in situ techniques have been used in the study of foaming evolution both from a chemical and a physical perspective. Infrared spectroscopy and small angle X-ray scattering (SAXS) studies have shown the effect of the added substances on the kinetics of polymerisation and matrix phaseseparated block copolymer structure development of PU foams. ${ }^{5,6,12-15}$ Meanwhile, both rheology $y^{5,14,16}$ and radioscopy ${ }^{17-19}$ have been used to analyse the foaming process from a physical perspective. In particular, previous studies of $\mathrm{PU}$ nanocomposite foams carried out, ${ }^{5,6}$ demonstrated the influence of carbon-based nanoparticles (CNPs), multi-walled carbon nanotubes (MWCNTs) and functionalised graphene sheets (f-GS), on the reaction kinetics and the development of the phase-separated morphology of flexible PU nanocomposite foams. These studies showed that the kinetics of polymerisation strongly depends on the morphology and surface bearing groups of the CNPs, delaying the extent of the reaction and affecting the micro-phase separated structure of PU foams. Studies on the foam structure evolution of PU nanocomposite foams have only recently been accomplished. The use of realtime X-ray monitoring has been developed as a suitable 
technique for the study of both polymer and metal foaming. Verdejo et al. ${ }^{19}$ studied the evolution of reactive polydimethylsiloxane (PDMS) foams reinforced with carbon nanotubes and graphene sheets by in situ synchrotron X-ray radioscopy. They reported differences in both the trend and speed of foaming and the dominating coarsening phenomena in the expansion and solidification processes. Pardo-Alonso et al. ${ }^{17,18}$ applied a similar X-ray technique, based on microfocus tube imaging, ${ }^{20}$ to observe the nucleation and expansion process of rigid PU nanocomposite foams. Thanks to the methodology developed it was possible to reach an excellent visualisation of the individual cells and thus a methodology to quantify the cell size and cell density during foaming was developed. Furthermore, the kinetics of density evolution could also be determined analysing the images obtained from the radiography sequences. These studies established suitability of this technique to quantify the effect of the nanofillers during the foaming process. Therefore, in the present work, the effects of carbonbased nanoparticles on the physical events taking place during the foaming evolution of flexible PU foams are studied, for the first time, by in situ microfocus X-ray radioscopy.

Present paper accomplishes a novel insight on the evolution of reactive $\mathrm{PU}$ nanocomposite systems by comparing the kinetics of blowing, polymerisation and cell structure degeneration mechanisms, which strongly influence the morphology and cellular structure of these systems and hence their final properties.

\section{Materials and methods}

\subsection{Flexible polyurethane (FPU) foams}

The polyol component used to prepare FPU foams was a highly reactive polyether-based triol, Voranol 6150 (Dow Plastics) with $\mathrm{OH}$ value of $27 \mathrm{mg} \mathrm{KOH}$ per $\mathrm{g}$, an average functionality of 3 and $M_{\mathrm{w}}$ of $6000 \mathrm{~g} \mathrm{~mol}^{-1}$ and a viscosity of $1150 \mathrm{mPa}$. The isocyanate was a methylene diphenyl diisocyanate (MDI), Voranate M2940 (Dow Plastics) with NCO content of 31.4\% and viscosity $49 \mathrm{mPa} \mathrm{s}^{-1}$ at $25{ }^{\circ} \mathrm{C}$. The polyether-based triol, Voranol CP1421 (Dow Plastics) with $\mathrm{OH}$ values of $31 \mathrm{mg} \mathrm{KOH}$ per $\mathrm{g}$ was used as a cell-opener in the reaction. The additives employed in this formulation were: FASCAT 4202 (dibutyl tin dilaurate from Arkema Inc.) which was used as a tin catalyst for the gelling reaction; TEDA L-33B (a solution of 33\% triethylendiamine in 1,4-butanediol from Torch Europe B.V.) and NIAX E-A-1 (a solution of $70 \%$ bis(2-dimethylaminoethyl) ether in $30 \mathrm{wt} \%$ dipropylene glycol from Momentive Performance Materials $\mathrm{Gmbh}$ ) as amine derivative catalysts in the blowing and gelling reactions, respectively; DEOA (85\% diethanolamine in water) as a cross-linker agent, $\mathrm{SH}-209$ (Momentive Performance Materials $\mathrm{Gmbh}$ ) as silicone surfactant and distilled water as blowing agent.

\subsection{Carbon based nanofillers}

Multi-walled carbon nanotubes (MWCNTs) were grown by the chemical vapor deposition (CVD) injection method based on the injection of a ferrocene (Aldrich 98\%)-toluene solution
(3 $\mathrm{wt} \%$ of ferrocene in toluene) at a constant rate in a tube furnace, under an inert argon atmosphere $\left(325 \mathrm{ml} \mathrm{min}^{-1}\right)$ and at a temperature of $760{ }^{\circ} \mathrm{C}$ in atmospheric pressure. ${ }^{21,22}$ These MWCNTs were chemically treated with a 3:1 concentrated sulfuric-nitric acid mixture and refluxed at $120{ }^{\circ} \mathrm{C}$ for $30 \mathrm{~min}$. Then, the mixture was filtered through a glass filter funnel using a PTFE membrane $(0.2 \mu \mathrm{m}$ pore size, Millipore) and washed with distilled water several times until neutral $\mathrm{pH}$. The oxidised MWCNTs (f-MWCNTs) were then dried at $120{ }^{\circ} \mathrm{C}$ and stored in a sealed container under vacuum prior to use to avoid possible effects of chemisorbed water due to their hydrophilic nature. ${ }^{23,24}$ The functional groups attached on the sidewalls of f-MWCNTs due to the oxidation are mainly hydroxyl and carboxyl groups. ${ }^{25}$

The synthesis of functionalised graphene sheets (f-GS) was carried out following different steps reported elsewhere. ${ }^{26}$ First, graphite oxide (GO) was produced using natural graphite (NG) powder (universal grade, 200 mesh, 99.9995\%, Fluka) according to the Brödie method. ${ }^{27,28}$ In a typical preparation procedure, a reaction flask with $20 \mathrm{ml}$ fuming nitric acid was cooled to $0{ }^{\circ} \mathrm{C}$ in a cryostat bath for $20 \mathrm{~min}$. Then, the graphite powder $(1 \mathrm{~g})$ was carefully added to avoid its agglomeration. After that, $\mathrm{KClO}_{3}(8 \mathrm{~g})$ was gradually added over $1 \mathrm{~h}$, in order to avoid sudden increases in temperature due to the exothermic nature of the reaction. The mixture was stirred for $21 \mathrm{~h}$ maintaining the reaction temperature at $0{ }^{\circ} \mathrm{C}$. Next, it was diluted in distilled water and filtered using a PTFE membrane until the filtrate reached a neutral $\mathrm{pH}$. The GO obtained was dried and stored in a vacuum oven at $50{ }^{\circ} \mathrm{C}$ until use. Then, the GO was thermally exfoliated at $1000{ }^{\circ} \mathrm{C}$ and under an inert argon atmosphere for a short time, usually less than $1 \mathrm{~min}$, to produce the f-GS. Finally, f-GS were stored in a sealed container prior to use. As produced f-GS contain epoxy, hydroxyl and carboxyl groups on their surface. ${ }^{25}$

\subsection{Preparation of FPU nanocomposite foams}

FPU foams were prepared from 100 phpp (parts per hundred parts of polyol) of Voranol 6150, 4 phpp of Voranol CP1421, 0.8 phpp of DEOA, 0.25 phpp of TEDA-L33B, 0.1 phpp of NIAX E-A1, 0.05 phpp of FASCAT, 0.4 phpp of silicone SH 209, 2 phpp of water and $43.4 \mathrm{phpp}$ of Voranate M2940. For comparison purposes, a fixed amount of nanofillers (0.5 phpp), 0.3 wt $\%$ in the final foam, was dispersed in the polyol (Voranol 6150). The mixture was initially sonicated for $10 \mathrm{~min}$ with an ultrasonication probe (Sonics VibraCell) in a water/ice bath, and was then stirred under high shear at $2400 \mathrm{rpm}$ for $6 \mathrm{~h}$. Subsequently, the surfactant, catalysts and distilled water were added to the polyol-nanofiller mixture and stirred at $2400 \mathrm{rpm}$ for $3 \mathrm{~min}$.

The foaming stage was promoted by stirring of the original polyol-nanofiller mixture and the additives with the isocyanate for 20 seconds at a speed of $2400 \mathrm{rpm}$. In order to delay/slow down the reaction rate the components were cooled to $5{ }^{\circ} \mathrm{C}$ prior to the liquid-liquid dispersion.

\subsection{Rheological characterisation}

The rheological behaviour of the polyol-nanofiller dispersions was measured using a TA Instruments Advanced Rheometer 
AR1000. The particular geometry used was a stainless-steel corrugated parallel plate with a diameter of $20 \mathrm{~mm}$. The gap was fixed to $0.3 \mathrm{~mm}$ and a dynamic frequency sweep from 0.01 to $100 \mathrm{rad} \mathrm{s}^{-1}$ at $21^{\circ} \mathrm{C}$ was employed.

\subsection{Time-resolved radiography}

The setup used for radiography acquisition with temporal resolution includes a microfocus X-ray source from Hamamatsu producing polychromatic X-rays and cone-beam geometry with a spot size 5-20 microns which allows up to 20 times magnification of the irradiated sample. ${ }^{20}$ The X-ray transmitted radiation is detected by a high sensitivity flat panel $(2240 \times 2344$ pixels, 12 bits and 50 microns pixel size) which allows high speed imaging up to $9 \mathrm{fps}$ (at $4 \times 4$ pixel binning). Finally a frame grabber records the radiography sequences for later image processing. The setup is customised for low absorbing materials such as polymers and typically works at low energies 20-60 kV and high currents $100-200 \mu \mathrm{A}$ in order to achieve an optimum contrast keeping small exposure times (600 ms, typically) and high acquisition rates (1.66 fps). Particularly, for this study the magnification used is 7.25 times with effective pixel size (after applying $2 \times 2$ binning) of 14 microns by using a source-detector distance of $580 \mathrm{~mm}$ and source-object distance of $80 \mathrm{~mm}$. A 3D drawing of the system is shown in Fig. 1.

For the radiographic experiments, a $0.6 \mathrm{~mm}$-thick stainless steel foaming frame was designed which allows observing only 2-3 cells in depth so they can be discerned by image analysis procedures. The frame has a circular cavity $(\varnothing=10 \mathrm{~mm})$ where a reacting droplet of FPU foam is placed just after stirring by a syringe for subsequent X-ray monitoring while foaming process takes place. Two evacuating conducts permitted to minimize movement of the expanding droplet improving the X-ray imaging in the central region of the cavity so cell growth can be visualised and further analysed. This very thin foaming frame was layered by two 250 microns-thick polypropylene (PP) films in order to keep the thickness unaltered during the entire observation.

\subsection{Image processing}

An image analysis procedure was needed in order to extract density and cell size information. The density evolution can be

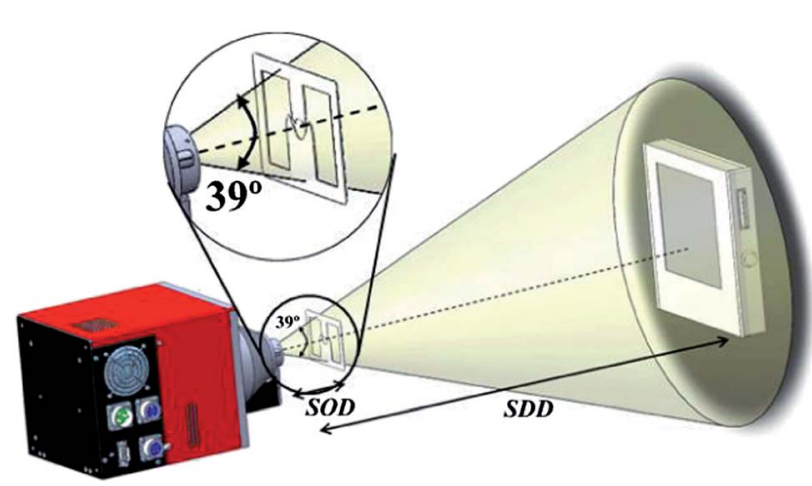

Fig. 1 Microfocus cone-beam radiography setup with including the foaming frame used. calculated from the radiograph intensities applying the BeerLambert law of attenuation (eqn (1)):

$$
I(x, z)=I_{0} \mathrm{e}^{-\mu \rho(x, z) d}
$$

where $\rho(x, z)$ is the density of the system, $d$ is the sample thickness in the beam direction assumed to be constant, $\mu$ is the attenuation coefficient and $I_{0}$ is the initial beam intensity. This equation is considered for monochromatic beam although remains still approximately valid if polychromatic rays are used $\left(\mu_{\text {eff }}\right)$. Furthermore, cell size was measured by an overlay method. ${ }^{29}$

\subsection{Scanning electron microscopy (SEM)}

The structure of the FPU nanocomposite foams was qualitatively examined using a Philips XL30 environmental scanning electron microscope (ESEM) at $15 \mathrm{kV}$. Cross-sections of the samples were cryo-fractured perpendicular to the foaming direction and the fracture surface was sputter-coated with gold/ palladium.

\section{Results and discussions}

\subsection{Rheological behaviour}

The formation of the cellular structure of FPU nanocomposite foams depends on different parameters such as viscosity of the initial polyol-CNP dispersions, surface tension and how the polymerisation reaction takes place. Hence, the study of the rheological behaviour of polyol-CNP systems is essential to understand the later foaming process on these foaming systems and it is influenced by the concentration of CNPs as well as their size, shape, presence or lack of functional groups, adsorption affinity of the polymer on the surface of the nanoparticles and the chain bridging between the nanofillers. ${ }^{30-35}$ Fig. 2 shows the dependence of the complex viscosity with the angular frequency for polyol and dispersions of 0.5 phpp of MWCNTs, f-MWCNTs and f-GS. It can be observed that measured values for neat

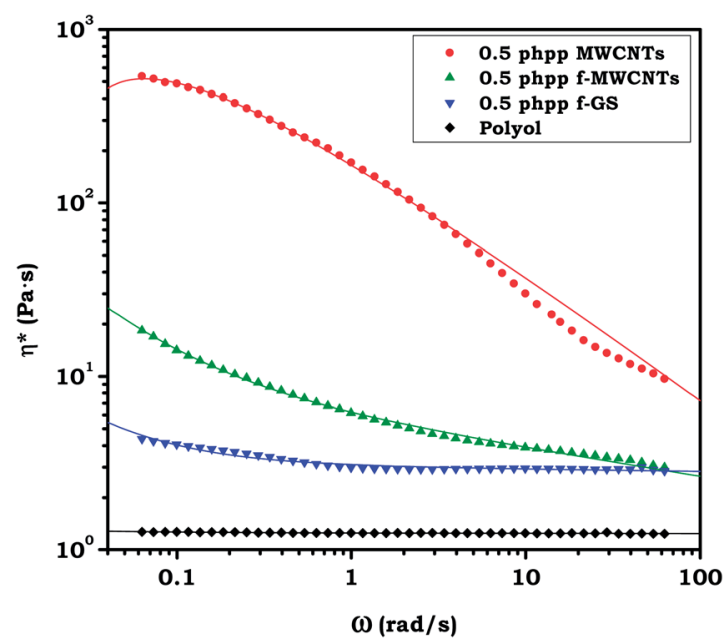

Fig. 2 Complex viscosity $\left(\eta^{*}\right)$ variation with the angular frequency $(\omega)$ of polyol-CNP dispersions. 
polyol are very similar to the ones given by the producer, i.e. $1150 \mathrm{mPa}$ s.

The Cox-Merz rule, that establishes a correlation between the steady state shear viscosity and the complex viscosity $(\eta(\omega)=\eta(\dot{\gamma})$ at $\omega=\dot{\gamma})$ has been described for uncured polymer nanocomposite systems and polymer nanocomposites filled with different types of nanofillers such as MWCNTs, carbon nanofibers among others at low concentrations. ${ }^{36-38}$ Therefore, the Cox-Merz rule could be validated in our study because of the fact that our suspensions are uncured polyol systems filled with low concentrations of carbon nanotubes and f-GS.

Then, the Herschel-Bulkley model for polyol-CNP dispersions described the rheological behaviour of these types of systems qualitatively, considering the abovementioned statements: $:^{5,39,40}$

$$
\eta^{*}=\frac{\tau_{0}}{\omega}+k \omega^{n-1}
$$

where $\eta^{*}$ is the complex viscosity, $\omega$ the angular frequency, $\tau_{0}$ the yield stress, $k$ the consistency index and $n$ is the flow behaviour index which describes the rheological behaviour (for $n<1$ shear-thinning, for $n>1$ shear-thickening and for $n=$ 1 Newtonian fluid). The parameters obtained from the fitting of the Herschel-Bulkley model are summarised in Table 1.

The results show that the polyol exhibits the typical behaviour of a Newtonian fluid, in which the viscosity is independent of the frequency. However, the polyol dispersions at $0.5 \mathrm{phpp}$ MWCNTs and f-MWCNTs show a shear-thinning behaviour since their complex viscosity reduces with shear rate while, their complex viscosity at low rates is 2 and 1 orders of magnitude higher than original polyol respectively. In the meanwhile, the polyol-f-GS dispersions exhibit still a quasi-linear behaviour at this concentration.

The dispersions of carbon nanotubes can be considered as rods in solution and, according to Doi and Edwards theory, ${ }^{\mathbf{4 1 , 4 2}}$ the rheology of rods in solution is strongly dependent on rod concentration and aspect ratio. The oxidation of the f-MWCNTs produces a less marked change of the complex viscosity compared to pristine MWCNTs, which is attributed to a shorter nanotube length and to the presence of functional groups, as a result of the aggressive acid treatment. ${ }^{31}$

The increase of the viscosity on polymer nanocomposites has been related to the formation of a nanoparticles network, via direct contacts or bridging by polymer chains. ${ }^{32,43}$ While polyolMWCNTs dispersions present a shear-thinning behaviour, dispersions with f-GS only show a weak increase of the viscosity compared to the neat polyol. This different behaviour is ascribed to their different morphology. MWCNTs are rod-shape

Table 1 Parameters and the correlation coefficient $\left(r^{2}\right)$ of the Herschel-Bulkley model for the polyol-CNP dispersions

\begin{tabular}{llccc}
\hline Sample & $\tau_{0}(\mathrm{~Pa})$ & $k\left(\mathrm{~Pa} \mathrm{~s}^{n}\right)$ & $n$ & $r^{2}$ \\
\hline Polyol & $\approx 0$ & 1.25 & 0.99 & 0.91 \\
0.5 phpp MWCNTs & 18.14 & 162.23 & 0.51 & 0.82 \\
0.5 phpp f-MWCNTs & 0.61 & 5.62 & 0.85 & 0.99 \\
0.5 phpp f-GS & 0.09 & 3.01 & 0.98 & 0.95
\end{tabular}

nanoparticles that facilitate interchain and entanglement interactions, and lead to a strong network as described by Knauert et al. ${ }^{32}$ and Pujari et al. ${ }^{44}$ Meanwhile, f-GS are considered as platelet-like nanoparticles with high aspect ratio and large interfacial areas, which in addition possess functional groups on their surface. Therefore, a great amount of molecules are adsorbed on the f-GS surface through hydrogen-bonding or electrostatic interactions, reducing the particle-particle interactions within the dispersions. ${ }^{32,45}$

\subsection{Density evolution}

The density evolution during foaming of FPU nanocomposite foams filled with CNPs has been studied by in situ X-ray radioscopy as described in the experimental section. Fig. 3 shows the evolution of the relative foam density. These results are plotted having as time reference the begging of stirring process and therefore all curves show a typical span of 50 seconds in the time scale (20 seconds mixing, droplet positioning, film covering and set up positioning). Therefore, in particular systems, the blowing reaction had already begun in the moment the monitoring started.

In Fig. 3 it can be appreciated a typical rapid density decrease at the early stages of the foaming process (50-100 s) due to the evolution of $\mathrm{CO}_{2}$ gas of the blowing reaction. After the nucleation stage, cells start to grow due to the gas diffusion from the higher-pressure regions (liquid phase) to the lower pressure ones (bubbles); meanwhile, the foam consolidates its structure due to the polymerisation reaction. Hence, its expansion rate slows down due to a combination of polymerisation (viscosity increase) evolution and the reduced amount of gas produced. The density evolution shows that the foams attain their final density after $400 \mathrm{~s}$, which coincides with the Berghmans point of these foams where vitrification freezes the morphology.,

The observed differences should mainly be ascribed to changes in the physical characteristics of the initial reactant, in particular the viscosity of the polyol-CNP dispersions, their surface tension and the interactions of the particles with the blowing reaction. The slope of the relative density (Fig. 3) at the initial stages (60-80 s) of the foaming is then related to these

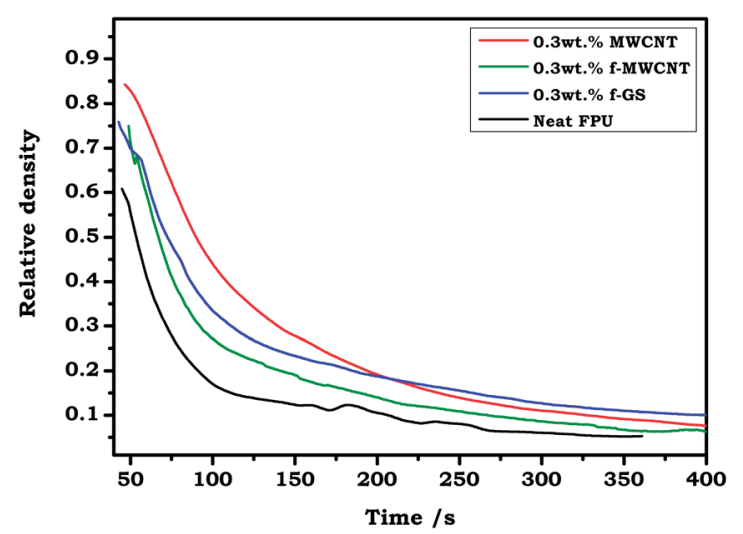

Fig. 3 Relative density evolution curves during foaming of FPU nanocomposite foams. 
Table 2 Values of the slope of the first points of relative density decrease of FPU nanocomposite foams

\begin{tabular}{ll}
\hline Sample & Slope $\left(-\% \mathrm{~s}^{-1}\right)$ \\
\hline Neat & 1.36 \\
MWCNTs & 0.89 \\
f-MWCNTs & 1.31 \\
f-GS & 1.08
\end{tabular}

characteristics and provides information about the foaming kinetics. The value of the slope (Table 2) decreases for nanocomposite foams containing CNPs, being the smallest for the system with MWCNTs followed by f-GS while the value for f-MWCNTs keeps practically unaltered. This observation seems to be, in part, related with the viscosity of the polyol - already determined in previous section - which agrees with a previous study by Bikard et al. ${ }^{46}$ showing that the larger the viscosity of the fluid, the slower the expansion in flexible foams. On the other hand, we can assume that the viscosity of the isocyanate is not having any significant influence considering it is 20 times lower than the one of the polyol. However, the obtained values do not directly correlate with the viscosity increase of the respective polyol-CNP dispersions since the chemical influence of CNPs on the reaction kinetics needs also to be taken into account. According to this, it seems that f-GS delay the reaction further than expected if we only consider the measured viscosity. Hence, the delay in the f-GS system should also respond to the presence of $\mathrm{C}-\mathrm{O}$ groups on these nanoparticles. Theoretical modelling has shown that these groups can create hydrogen bonds with adsorbed water on their surface. ${ }^{47}$ Consequently, the number of water molecules available for the reaction with the isocyanate is reduced with respect to the other systems and thus the generation of $\mathrm{CO}_{2}$ gas, in agreement with previous results by in situ FTIR and synchrotron SAXS. ${ }^{6}$ In the case of MWCNT's nanocomposite foam a stronger effect related to the mobility of these systems because of their rigidity and their obstruction on the formation of hard segments during the polymerisation, needs to be consider as regards of the results observed in bubble nucleation in next section. ${ }^{6}$

Besides the delay in the density evolution characterised throughout all the stages of the reaction, the final density of the FPU nanocomposite foams is slightly higher than neat PU foam, such increase is obviously attributed to a reduced volume expansion with no effect of the intrinsic density of the CNPs. This expansion reduction has also been observed in previous studies of CNPs filled PU foams. ${ }^{24}$ The density increase is attributed to the viscosity increase of the mixtures, the wetting character of the CNPs, which in turn affect the matrix phaseseparated morphology, and the mentioned chemical interaction, which reduces the amount of water molecules available.

\subsection{Cell size evolution}

The acquired radiographies of FPU nanocomposite foams at three different times can be observed in Fig. 4 in comparison to neat FPU foams. It is important to keep in mind that cell size a)

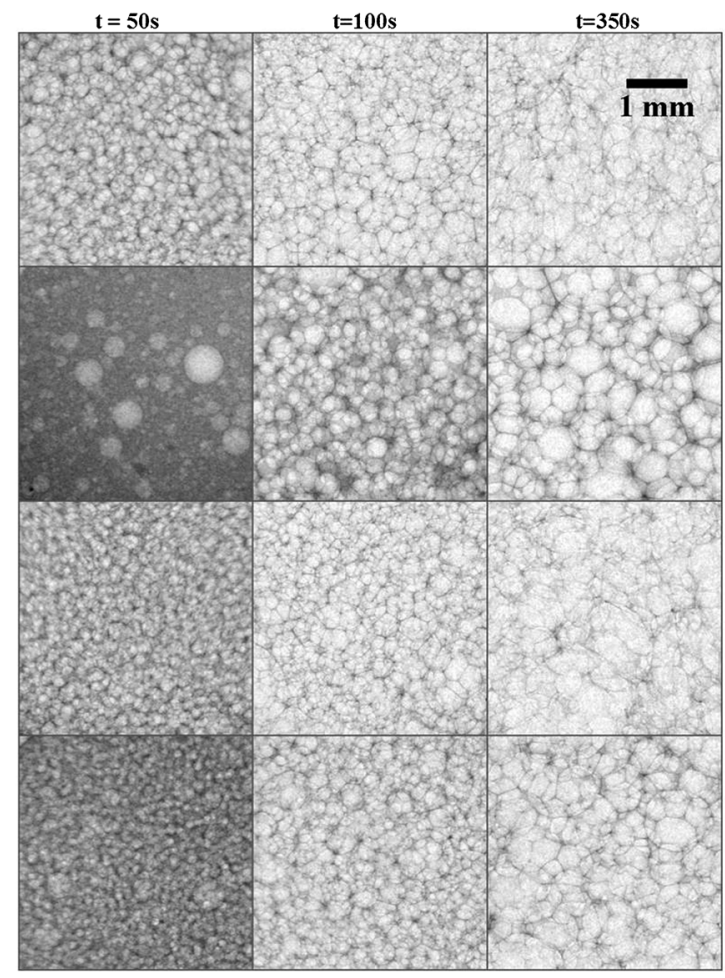

Fig. 4 X-ray radiographs of expanding flexible $P U$ nanocomposite foams for: (a) neat, (b) MWCNTs, (c) f-MWCNTs and (d) f-GS.

evolution of PU depends on both the continuously polymerising liquid matrix and the amount of dispersed gas phase, having several physico-chemical factors contributing to them. ${ }^{4}$ The simple observation of the images demonstrates that the cell size of the open-cell FPU nanocomposite foams is smaller, at initial foaming stages, than the neat formulation. Nevertheless, this first evaluation is still not completely clarifying since the images, at a same instant, correspond to different expansion grades and thus some corrections need to be considered. On the other hand, in this first approach, CNPs appear to act as nucleating agents in flexible PU foams similar to the results observed for rigid foams with the same CNPs used in this study $^{\mathbf{4 0}}$ and filled with nanoclays or nanosilicas. ${ }^{17}$

It can also be observed that final state $(t=350 \mathrm{~s})$ of the produced nanocomposite foams does not strictly correspond to the cell size reduction observed in the initial stage, which in principle implies the presence of any degeneration mechanism during foam evolution. Cell diameters at this stage seem to agree with the cell size obtained in SEM micrographs Fig. 6.

The results for the quantitative analysis of cell size evolution are given in Fig. 5. This analysis excludes from the average diameter characterisation the larger pores shown in the images - actually air entrapments - such as the ones clearly visualised in Fig. $4 \mathrm{~b}$ at $t=50 \mathrm{~s}$. The graph in Fig. 5 shows a dissimilar poregrowing slope for the different analysed materials. In our particular case, the slope is much higher in the case of MWCNTs followed by f-GSs and f-MWCNTs with a rather lower value in the case of neat PU. The quicker transition from smaller to larger pores can be interpreted as a sign of cell instability, i.e. 


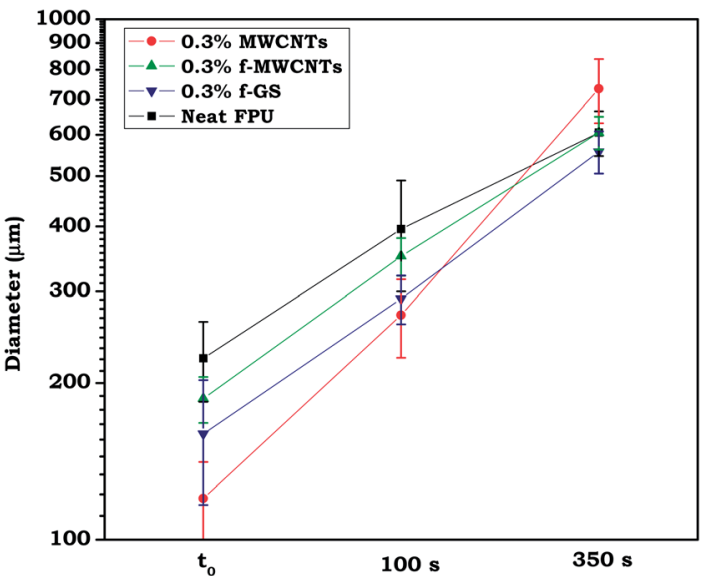

Fig. 5 Diameter of expanding flexible PU nanocomposite foams at 50, $100 \mathrm{~s}$ and $350 \mathrm{~s}$.

presence of coalescence, although the different dynamics in density evolution also needs to be taken into account. However, the cell size on its own could not completely elucidate the events occurring while the foaming process is taken place and it is used in combination with the cell density evolution to explain the different phenomena.

\subsection{Cell density evolution}

Cell density values were calculated according to eqn (3) and exhibit a varying behaviour depending on the nanocomposite formulation used (Fig. 6). According to the results, it is possible to assess that neat formulation does not show a significant cell density decrease (at least upon the analysed point), while the nanocomposite formulations seem to promote instability in the evolving foam. Hence, flexible PU foam is largely affected by the presence of both carbon nanotubes and functionalised graphene sheets while the functionalised nanotubes present a smaller interaction. Previous studies on rigid PU nanocomposite closed cell foams reinforced with organoclays

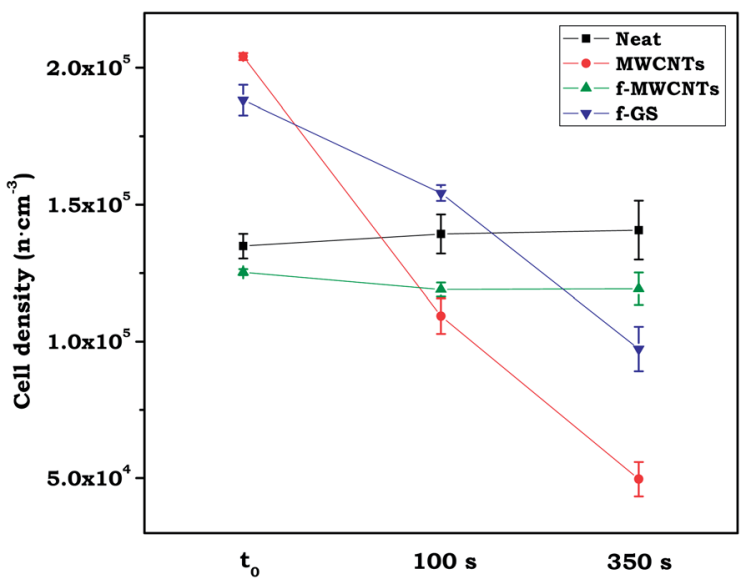

Fig. 6 Cell density values of FPU nanocomposite foams at $t_{0}, 100 \mathrm{~s}$ and $350 \mathrm{~s}$. showed a constant cell density throughout all the foaming process without film ruptures. ${ }^{17,18}$ Although in the particular formulations of open cell foams the surfactant content is finely adjusted in order to prevent coalescence upon an optimum viscosity, which subsequently allows cell opening via cell wall thinning but generally prevents coalescence. The results also confirm the apparent nucleation effect observed at the early stages (Fig. 5) both for MWCNT and f-GS nanocomposite foams. In the case of MWCNT the nucleation enhancement reaches $85 \%$ whereas in the case of f-GS we can observe a $30 \%$ and in the case of f-MWCNT the nucleation is similar.

$$
N_{\mathrm{C}}=\frac{6}{\pi \phi^{3}}\left(\frac{\rho_{\text {solid }}}{\rho_{\text {foam }}}-1\right)
$$

The nucleation mechanism mainly depends on the interfacial surface area between the polymer and the nanoparticles and, obviously, on their dispersion and surface character. ${ }^{3}$ Therefore, the addition of nanofillers induces heterogeneous nucleation in polymer foams, being their efficiency higher as their dispersion and distribution in the polymer matrix are optimal. Nonetheless, one of the main aspects that should be taking into consideration on the effectiveness of the nucleation is the surface nature of the nanoparticles besides their surface morphology. The inclusion of oxygen-bearing groups during the functionalisation of carbon nanotubes and graphene sheets changes the hydrophobic character of these CNPs to hydrophilic. Highly hydrophobic nanofillers, such as MWCNTs, favoured the nucleation mechanism due to their non-wetting surface, inducing destabilizing effects in the thin membranes at longer times via the so-called bridging-dewetting mechanisms. ${ }^{48-50}$ Meanwhile, hydrophilic nanoparticles, as f-MWCNTs, have the opposite effect and appear to act as foam stabilizers. In this context, it should be expected for f-GS similar behaviour as for f-MWCNTs because of their hydrophilic character thanks to the presence of epoxy, hydroxyl and carboxyl groups. However, systems with f-GS present an intermediate state between MWCNTs and f-MWCNTs. Hence, the flat surface of graphene plays a key role on the initial nucleation mechanism, also observed in nanofillers with the same morphology i.e. nanoclays ${ }^{17}$ but their hydrophilic nature avoid the bridgingdewetting mechanisms observed on MWCNTs.

With regards the coalescence phenomena we can assess from the numerical results and the visual observation of the process, that MWCNTs induce the largest coalescence throughout the full expansion process. On the other hand, fMWCNTs show a no appreciable cell density reduction keeping a nearly constant value and, indeed, coalescence is not observed in the process. Finally, a similar strong effect on bubble stability is observed in the case of f-GS with a cell density decaying more than $100 \%$ of the initial value. These differences could be, in part, attributed to the surface nature of the nanoparticles mentioned above and their effect on the phase-separated matrix morphology. Nevertheless, other effects such as the interaction of these particles with the urea hard segments during the gelling reaction need to be considered. In particular, for the system showing a strong coalescence (MWCNT's 

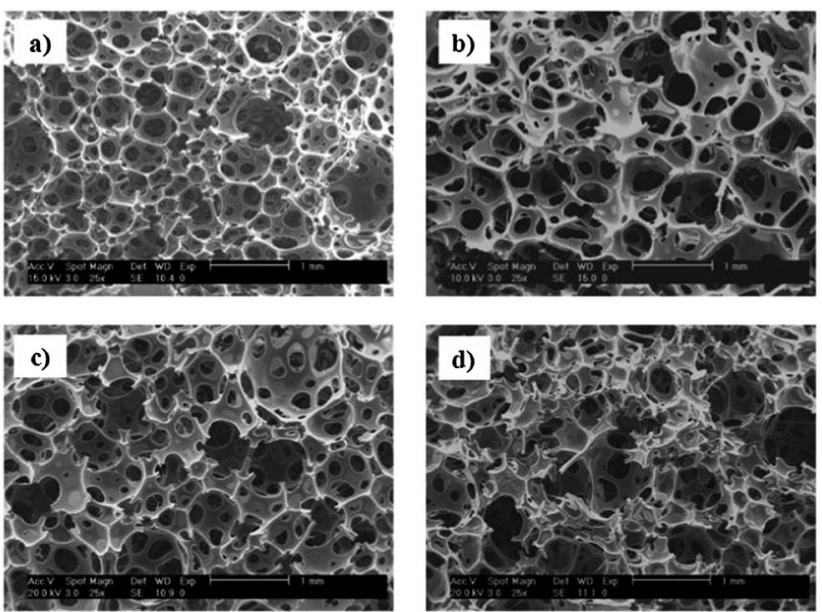

Fig. 7 Representative SEM images of flexible PU nanocomposite foams: (a) neat, (b) MWCNTs, (c) f-MWCNTs and (d) f-GS

nanocomposite foam), the formation of denser urea aggregates, observed by AFM, ${ }^{6}$ forms a greater phase separation but as a consequence increases the intensity of the coalescence phenomena.

Micrographs in Fig. 7 show that results obtained via in situ X-ray radioscopy are consistent with the ex situ results of produced and stabilised foams. The results reveal that final cell size in the case of both types of MWCNTs is larger than the one observed in the neat formulation. On the other hand the cell size for f-GS is little smaller although the quality of the micrograph does not allow to clearly evaluate it. It is important to mention that the foam matrix with f-GS seems to reach uncompleted curing, which causes structure deterioration during sample preparation.

\section{Conclusions}

The foaming evolution of flexible PU nanocomposite foams has been examined by in situ X-ray radioscopy and the effects of carbon-based nanofillers and their surface treatment have been observed. The rheological behaviour of the polyol-CNP dispersions is influenced by the nanoparticles, particularly in the case of MWCNTs. Expansion kinetics is delayed by the CNP, probably due to a combination of enhanced viscosity (physical interaction) and a delay in the blowing kinetics (chemical interaction). The results at early stages also indicate that there is a significant cell nucleation enhancement for MWCNT and the f-GS nanocomposite foams. Only the f-MWCNT's seem to promote a similar number of bubbles that neat FPU. On the other hand, the surface nature of the carbon nanoparticles, hydrophilic or hydrophobic, influences the physical events taking place on the evolved reactive PU nanocomposite foams, which corroborates the chemical processes observed for these systems by in situ FTIR and synchrotron SAXS. Cell size evolution is strongly determined by the chemical interaction of the FPU formulation with the CNP added. Curiously, those particles that actively promoted the nucleation, due to a bridging-dewetting mechanism and surface morphology, seem to form denser urea hard domains during the gelling reaction promoting coalescence that drives to final lower cell densities.

\section{Acknowledgements}

Financial support from the Spanish Ministry of Science and Innovation and FEDER funds (MAT2009-14001-C02-01, MAT 2010-18749 and MAT 2012-34901), the Junta of Castille and Leon (project VA035U13), the European Space Agency (Project MAP AO-99-075), the $7^{\text {th }}$ Framework Program of E.U. through HARCANA (NMP3-LA-2008-213277), Juan de la Cierva contract by the Ministry of Economy and Competitiveness (E. Solórzano, JCI-2011-09775) and PIRTU contract by Junta of Castile and León co-financed by the European Social Fund (S. Pardo-Alonso, EDU/289/2011) are gratefully acknowledged. M. M. Bernal also acknowledges the FPI from MICINN.

\section{References}

1 L. J. Lee, C. Zeng, X. Cao, X. Han, J. Shen and G. Xu, Compos. Sci. Technol., 2005, 65, 2344-2363.

2 C. C. Ibeh and M. Bubacz, J. Cell. Plast., 2008, 44, 493-515.

3 L. Chen, D. Rende, L. S. Schadler and R. Ozisik, J. Mater. Chem. A, 2013, 1, 3837-3850.

4 G. Harikrishnan, T. U. Patro, A. R. Unni and D. V. Khakhar, Soft Matter, 2011, 7, 6801-6804.

5 M. M. Bernal, M. A. Lopez-Manchado and R. Verdejo, Macromol. Chem. Phys., 2011, 212, 971-979.

6 M. M. Bernal, M. Martin-Gallego, L. J. Romasanta, A.-C. Mortamet, M. A. López-Manchado, A. J. Ryan and R. Verdejo, Polymer, 2012, 53, 4025-4032.

7 G. Woods, Flexible Polyurethane Foams. Chemistry and Technology, Applied Science Publishers LTD, London, 1982.

8 D. Klempner and V. Sendijarevic, Handbook of polymeric foams and foam technology, Hanser Publishers, Munich, 2004.

9 S. T. Lee and N. S. Ramesh, Polymeric foams: mechanisms and materials, CRC Press LLC, Boca Raton, Florida, USA, 2004.

10 J. Bicerano, R. D. Daussin, M. J. A. Elwell, H. R. v. d. Wal, P. Berthevas, M. Brown, F. Casati, W. Farrissey, J. Fosnaugh, R. d. Genova, R. Herrington, J. Hicks, K. Hinze, K. Hock, D. Hunter, L. Jeng, D. Laycock, W. Lidy, H. Mispreuve, R. Moore, L. Nafziger, M. Norton, D. Parrish, R. Priester, K. Skaggs, L. Stahler, F. Sweet, R. Thomas, R. Turner, G. Wiltz, T. Woods, C. P. Christenson and A. K. Schrock, in Polymeric Foams. Mechanisms and Materials, ed. S. T. Lee and N. S. Ramesh, CRC Press, Boca Raton, Florida, USA, 2004.

11 R. A. Neff and C. W. Macosko, Rheol. Acta, 1996, 35, 656-666. 12 G. Harikrishnan, T. U. Patro and D. V. Khakhar, Ind. Eng. Chem. Res., 2006, 45, 7126-7134.

13 A. N. Wilkinson, N. H. Fithriyah, J. L. Stanford and D. Suckley, Macromol. Symp., 2007, 256, 65-72.

14 W. Li, A. J. Ryan and I. K. Meier, Macromolecules, 2002, 35, 5034-5042. 
15 M. J. Elwell, A. J. Ryan, H. J. M. Grünbauer and H. C. Van Lieshout, Macromolecules, 1996, 29, 2960-2968.

16 X. D. Zhang, D. W. Giles, V. H. Barocas, K. Yasunaga and C. W. Macosko, J. Rheol., 1998, 42, 871-889.

17 S. Pardo-Alonso, E. Solorzano, S. Estravis, M. A. RodriguezPerez and J. A. de Saja, Soft Matter, 2012, 8, 11262-11270.

18 S. Pardo-Alonso, E. Solórzano and M. A. Rodriguez-Perez, Colloids Surf., A, 2013, 438, 119-125.

19 R. Verdejo, F. J. Tapiador, L. Helfen, M. M. Bernal, N. Bitinis and M. A. Lopez-Manchado, Phys. Chem. Chem. Phys., 2009, 11, 10860-10866.

20 E. Solórzano, J. Pinto, S. Pardo, F. Garcia-Moreno and M. A. Rodriguez-Perez, Polym. Test., 2013, 32, 321-329.

21 C. Singh, M. S. Shaffer and A. H. Windle, Carbon, 2003, 41, 359-368.

22 P. J. F. Harris, Carbon nanotube science. Synthesis, properties and applications, Cambridge University Press, 2009.

23 R. Verdejo, S. Lamoriniere, B. Cottam, A. Bismarck and M. Shaffer, Chem. Commun., 2007, 513-515.

24 R. Verdejo, R. Stampfli, M. Alvarez-Lainez, S. Mourad, M. A. Rodriguez-Perez, P. A. Bruhwiler and M. Shaffer, Compos. Sci. Technol., 2009, 69, 1564-1569.

25 M. M. Bernal, Study of polyurethane nanocomposite foams reinforced with carbon-based nanofillers, Universidad Politécnica de Valencia, 2012.

26 R. Verdejo, F. Barroso-Bujans, M. A. Rodriguez-Perez, J. A. de Saja and M. A. Lopez-Manchado, J. Mater. Chem., 2008, 18, 2221-2226.

27 B. C. Brödie, Philos. Trans. R. Soc. London, 1859, 149, 249259.

28 D. R. Dreyer, S. Park, C. W. Bielawski and R. S. Ruoff, Chem. Soc. Rev., 2010, 39, 228-240.

29 E. Solórzano, S. Pardo-Alonso, J. A. d. Saja and M. A. Rodriguez-Perez, Colloids Surf., A, 2013, 438, 167-173.

30 P. Potschke, T. D. Fornes and D. R. Paul, Polymer, 2002, 43, 3247-3255.

31 J. H. Xu, S. Chatterjee, K. W. Koelling, Y. R. Wang and S. E. Bechtel, Rheol. Acta, 2005, 44, 537-562.
32 S. T. Knauert, J. F. Douglas and F. W. Starr, J. Polym. Sci., Part B: Polym. Phys., 2007, 45, 1882-1897.

33 T. Ramanathan, A. A. Abdala, S. Stankovich, D. A. Dikin, M. Herrera-Alonso, R. D. Piner, D. H. Adamson, H. C. Schniepp, X. Chen, R. S. Ruoff, S. T. Nguyen, I. A. Aksay, R. K. Prud'homme and L. C. Brinson, Nat. Nanotechnol., 2008, 3, 327-331.

34 H. Kim, A. A. Abdala and C. W. Macosko, Macromolecules, 2010, 43, 6515-6530.

35 D. R. Heine, M. K. Petersen and G. S. Grest, J. Chem. Phys., 2010, 132, 184509.

36 K. L. White, P. Li, Y. Sumi and H.-J. Sue, J. Phys. Chem. B, 2013, 118, 362-371.

37 J. Xu, S. Chatterjee, K. Koelling, Y. Wang and S. Bechtel, Rheol. Acta, 2005, 44, 537-562.

38 H. Song, J. Zhang, Y. Niu and Z. Wang, J. Phys. Chem. B, 2010, 114, 6006-6013.

39 H. S. Xia and M. Song, Soft Matter, 2005, 1, 386-394.

40 M. M. Bernal, M. Martin-Gallego, I. Molenberg, I. Huynen, M. A. Lopez Manchado and R. Verdejo, RSC Adv., 2014, 4, 7911-7918.

41 M. Doi and S. F. Edwards, The Theory of Polymer Dynamics, Oxford Press, London, 1986.

42 S. Marceau, P. Dubois, R. Fulchiron and P. Cassagnau, Macromolecules, 2009, 42, 1433-1438.

43 H. Kim and C. W. Macosko, Polymer, 2009, 50, 3797-3809.

44 S. Pujari, S. Rahatekar, J. W. Gilman, K. K. Koziol, A. H. Windle and W. R. Burghardt, J. Rheol., 2011, 55, 1033-1058.

45 C. Wan and B. Chen, J. Mater. Chem., 2012, 22, 3637-3646.

46 J. Bikard, J. Bruchon, T. Coupez and B. Vergnes, J. Mater. Sci., 2005, 40, 5875-5881.

47 J. Berashevich and T. Chakraborty, Phys. Rev. B: Condens. Matter Mater. Phys., 2010, 81, 205431.

48 R. G. Alargova, D. S. Warhadpande, V. N. Paunov and O. D. Velev, Langmuir, 2004, 20, 10371-10374.

49 B. P. Binks, Curr. Opin. Colloid Interface Sci., 2002, 7, 21-41. 50 R. Aveyard, B. P. Binks, P. D. I. Fletcher, T. G. Peck and C. E. Rutherford, Adv. Colloid Interface Sci., 1994, 48, 93-120. 